\title{
Measurements of forest structures by combining different point cloud data
}

\author{
Wang Tao \\ Guangzhou Institute of Geography, China \\ wangtao8869@163.com
}

\begin{abstract}
Keywords: LiDAR; multi-view dense match point cloud; Forest structural parameters extraction
Abstract. It is useful to monitor forests by extracting parameters from LiDAR and aerial imagery. Reconstructing them can be challenging using only LiDAR because it is difficult to extract exact parameters. Therefore, using aerial images, we can obtain a digital surface model by matching, extract a digital terrain model by filtering, and generate a canopy height model(). In forests, we obtain a point cloud from stereo images by using a multi-view dense matching algorithm, and then fuse the results to generate a high-density digital surface model. Finally, we can extract the plant information by combining the LiDAR and matched point cloud.
\end{abstract}

\section{Introduction}

Point clouds are widely used in forest parameter extraction. However, they are usually generated using LiDAR, so have many disadvantages. In forest investigation, it is difficult to obtain a high-density point cloud (Leverl et al., 2010).

The objective of this paper is to evaluate the quality, accuracy, and feasibility of an automatic tree extraction method based on low density LiDAR point data and a dense match point cloud.

The European Spatial Data Research Organization started a benchmark on image based digital surface model (DSM) generation in February 2013. This test is based on two representative image blocks, which were processed by different groups with different software systems.

The interpretation of the benchmark results identified some scenarios that still can cause some problems during image based surface reconstruction. Some solutions showed decreasing accuracies as shadows were cast. In addition, the representative image blocks don't include the forest area (Haala, 2013).

Dense matching methods can be divided into two types: depth map fusion and object patch. One technique used to reduce the search area of matching processes in aerial images is MVLL (Multi-View Local Learning), which defines a vertical line in the object space and calculates the correlation coefficients of the two image matrices according to a point along this line (Zhang, 2005; Waser, 2008; Baltsavias, 2008; Jiang, 2004; Ming, 2009). Another method is PMVS (Patch-based Multi-View Stereo), which is a patch-based method. This method is implemented as a match, expand, and filter procedure, starting from a sparse set of matched key points, and repeatedly expanding these to nearby pixel correspondences before using visibility constraints to filter away false matches (Zhu, 2009; Furukawa, 2008; Furukawa and Jean, 2009; Furukawa et al., 2009b; Shi, Guo and Hu, 2011).

Inter-scanline inconsistencies can be resolved by techniques, which use two-dimensional constraints, like graph cut or belief propagation stereo analysis.

SGM (Semi-Global Matching), published by H.Hirschmuller in 2005, approximates global optimization by combining multiple one-dimensional optimization criteria.

Juha Hyyppa (2008) used small footprint laser scanning data to investigate the forest. This method generates a digital model first, filters the normalized digital terrain model, determines the local maximum, and then uses the growing method to segment the tree crown.

The percentage of detected trees varied between 25 to $90 \%$, implying different capabilities in detecting suppressed trees. The best models were significantly better in separating tree groups into individual trees compared to the manual method. Surprisingly, there was no improvement in the detection rate when the pulse density was increased from 2 to $8 \mathrm{pt} / \mathrm{m}^{2}$ (Shan and Charles, 2008). 


\section{Methods}

This method includes accurate image orientation, DSM extraction, and tree parameter extraction. After the images were captured, they were matched to generate connection points. A bundle block adjustment was then used to obtain the accurate orientation parameter. LiDAR data were filtered to obtain a digital elevation model (DEM). The DSM was generated using dense matching. Then, the DSM and DEM were used to generate the CHM. After segmenting the CHM, then a local maximum algorithm was used to find the location and height of the trees.

Under the forest area, it is difficult to set control points. Therefore, we obtained the image orientation parameters by using a GPS-supported bundle adjustment, then a multi-view dense match to generate the dense matched point cloud. The DEM was generated by filtering the LiDAR data.

We used the calibrated bundle adjustment to obtain the orientation and camera parameters using the basic model shown in equation (1):

$$
\begin{aligned}
& x-x_{0}-\Delta x=-f \frac{a_{1}\left(X-X_{s}\right)+b_{1}\left(Y-Y_{s}\right)+c_{1}\left(Z-Z_{s}\right)}{a_{3}\left(X-X_{s}\right)+b_{3}\left(Y-Y_{s}\right)+c_{3}\left(Z-Z_{s}\right)}=-f \frac{\bar{X}}{\bar{Z}} \\
& y-y_{0}-\Delta y=-f \frac{a_{2}\left(X-X_{s}\right)+b_{2}\left(Y-Y_{s}\right)+c_{2}\left(Z-Z_{s}\right)}{a_{3}\left(X-X_{s}\right)+b_{3}\left(Y-Y_{s}\right)+c_{3}\left(Z-Z_{s}\right)}=-f \frac{\bar{Y}}{\bar{Z}}
\end{aligned}
$$

Where $\Delta x$ and $\Delta y$ are as shown in equation (2):

$$
\begin{aligned}
& \Delta x=\left(x-x_{0}\right)\left(K_{1} r^{2}+K_{2} r^{4}+K_{3} r^{6}\right)+P_{1}\left(r^{2}+2\left(x-x_{0}\right)^{2}\right)+2 P_{2}\left(x-x_{0}\right)\left(y-y_{0}\right) \\
& \Delta y=\left(y-y_{0}\right)\left(K_{1} r^{2}+K_{2} r^{4}+K_{3} r^{6}\right)+P_{2}\left(r^{2}+2\left(y-y_{0}\right)^{2}\right)+2 P_{1}\left(x-x_{0}\right)\left(y-y_{0}\right)
\end{aligned}
$$

Where $K_{1}, K_{2}$, and $K_{3}$ are radial factors, and $P_{1}$ and $P_{2}$ are tangential factors.

We used a SURE(Photogrammetric Surface Reconstruction from Imagery) algorithm to generate the dense matched point cloud. It applied the SGM algorithm to match between image pairs, and then fused the results to obtain the resulting point clouds. The SGM algorithm aims to estimate disparities across stereo pairs such that the global cost function shown in equation (3) is minimized.

$$
E(\mathrm{D})=\sum_{x_{b}}\left(C\left(\mathrm{x}_{b}, \mathrm{D}\left(\mathrm{x}_{b}\right)\right)\right)+\sum_{x_{N}} P_{1} T\left[\left\|\mathrm{D}\left(\mathrm{x}_{b}\right)-\mathrm{D}\left(\mathrm{x}_{N}\right)\right\|=1\right]+\sum_{x_{N}} P_{2} T\left[\left\|\mathrm{D}\left(\mathrm{x}_{b}\right)-\mathrm{D}\left(\mathrm{x}_{N}\right)\right\|>1\right]
$$

In this equation $\mathrm{D}$ represents the disparity image holding estimates of all base image pixels $\mathrm{x}_{b}$. $T$ is an operator that evaluates whether the subsequent condition is true and is set to zero if false. $\mathrm{x}_{N}$ denotes the base image pixels in the neighborhood of $\mathrm{x}_{b}$. The global cost function, $E$, is composed of a data term and two terms representing smooth surfaces. The data term is computed using pixel-wise similarity measures $C\left(\mathrm{x}_{b}, \mathrm{x}_{m}\right)$. The penalty parameters, $P_{1}$ and $P_{2}$, control the gain of surface smoothing (Rothermel et al., 2011; Rothermel et al., 2012; Wenzel et al., 2013).

Progressive densification was used for filtering. The entire point dataset was divided into tiles, and then the lowest points in each tile were used as initial ground points. Then a triangular irregular network (TIN) of those ground points was constructed as the reference surface. For each triangle, one of the still unclassified points inside was added to the set of ground points if the distance from the point to the TIN facet and the angle between the TIN facet and the line connecting the point with the facet's closest vertex did not exceed given thresholds.

The method then uses a CHM to identify local maxima using a variable size evaluation window. The window size is based on the canopy height, and is determined by the height of the surface at the center of the window using the following equation (4):

$$
\text { width }=A+B^{*} h t+C^{*} h t^{2}+D^{*} h t^{3}+E^{*} h t^{4}+F^{*} h t^{5}
$$


Coefficients are calculated by using locally obtained tree height and crown width measurements.

\section{Results}

The desity of the matched point cloud was 65 points $/ \mathrm{m}^{2}$. The density of the LiDAR measurements was 3-4 points $/ \mathrm{m}^{2}$. In the test area, there were many control points, and they were used to check the accuracy of the DEM of the LiDAR. The height of the trees were used to check the accuracy of the LiDAR and matched point cloud. The avgabs (average of the absolute value of the error), rms (route mean square), stddev (standard deviation), and avg (average) of the LiDAR were 8.4986, 9.58415, 4.45425, and 8.487, respectively. The avgabs, rms, stddev, and avg of the matched point cloud were $10.5831,10.9816,2.93263$, and 10.5831, respectively. Percentage of the extraction was $30 \%$.

\section{Discussion}

Airborne LiDAR data are often used to provide detailed information on tree canopy structure. Lower accuracy was obtained when using low density LiDAR data, as it is difficult to capture the crown shape using this method. By using the dense match method, it is possible to get a dense point cloud. However, this process is constrained by difficulties in the dense match method. In this paper, SGM algorithm was used to match image pairs, and then the result was fused to get a more accurate and dense point cloud, with a result of 65 points $/ m^{2}$.

Although we have demonstrated that combining data from a dense matched point cloud and LiDAR can make up for the limitation of a low density LiDAR point cloud, the extraction result is not as good as the dense point cloud. Additional research is needed to explore additional dense match and extraction methods.

\section{Conclusions}

A method to extract forest tree parameters by integrating LiDAR and multi-view dense matched point cloud data is described here. The SURE dense match method was used to generate a point cloud, which is denser than LiDAR data. Progressive densification was used to generate the DEM.

These results show it is useful to combine the multi-view dense matched point cloud and LiDAR data to extract forest tree parameters.

\section{Acknowledgements}

Data for this work were provided by the Key Project of Chinese National Programs for Fundamental Research and Development (973 program No. 2007CB714404), the Knowledge Innovation Program of the Chinese Academy of Sciences (kzcx2-YW-313) and the National Natural Science Foundation of China (grant no. 40730525 and 40971203).

\section{References}

[1]Baltsavias, E., Gruen, A., Eisenbeiss,H., Zhang,L. and Waser, L. T., 2008. "High-quality image matching and automated generation of 3D tree models." International Journal of Remote Sensing 29 (5), 1243-1259.

[2]Furukawa Yasutaka, 2008. "High-Fidelity IMAGE-BASED MODELING.” Ph D. diss., University of Illinois.

[3]Furukawa Yasutaka and Jean Ponce, 2009. "Accurate, Dense, and Robust Multi-View Stereopsis." IEEE Transactions on Pattern Analysis and Machine Intelligence. 
[4]Furukawa Yasutaka, Brian Curless, Steven M. Seitz, and Richard Szeliski, 2009. "Reconstructing Building Interiors from Images." paper presented at International Conference on Computer Vision.

[5]Haala, N., 2013. "The Landscape of Dense Image Matching Algorithms." paper presented at Photogrammetric Week 2013, Wichmann Verlag, Berlin/Offenbach.

[6]Hirschmüller, H., 2008. "Stereo Processing by Semiglobal Matching and Mutual Information." IEEE Transactions on Pattern Analysis and Machine Intelligence 30, 328-341.

[7]Jiang Wanshou, 2004. "Multiple Aerial Image Matching and Automatic Building Detection.” Ph D. diss., University of Wuhan.

[8]Leberl F, A. Irschara, T. Pock, P. Meixner, M. Gruber, S. Scholz, and A. Wiechert, 2011. "Point Clouds: Lidar versus 3D Vision.” Photogrammetric \& Remote Sensing 76 (10): 1123-1134.

[9]Liu Hongxin, 2011. "Research on algorithm of stereo correspondence based on graph cut." Dissertation. University of Hunan.

[10]Liu Yingjie, 2011. "Research on stereo matching algorithm based on dynamic programming and belief propagation.” Dissertation. Yanshan University of Yanshan.

[11]Ming Yang, 2009. "Research on Key Technologies of Automatic Image Matching for Special Aerial Images.” Ph D. diss., University of Wuhan.

[12]Rothermel, M., Wenzel, K., Fritsch, D., Haala, N, 2012. "SURE: Photogrammetric Surface Reconstruction from Imagery." paper presented at Proceedings LC3D Workshop, Berlin. December.

[13]Rothermel, M., Haala, N., 2011. "Potential of Dense Matching for the Generation of High Quality Digital Elevation Models.” paper presented In ISPRS Proceedings XXXVII 4-W19.

[14]Shi Limin, Guo Fusheng, Hu Zhanyi, 2011. "An Improved PMVS through Scene Geometric Information." ACTA AUTUMATICA SINUCA 37 (5): 560-568.

[15]Szeliski, Richard, 2011. Computer Vision: Algorithm and Applications. Springer.

[16]Shan Jie, Charles K.Toth, 2008. Topographic laser ranging and scanning principles and processing. CRC press.

[17]Waser, L.T., Baltsavias, E., Ecker, K., Eisenbeiss, H., Ginzler, C., Küchler, M., Thee, P. and Zhang, L. 2008. "High-resolution digital surface models (DSMs) for modeling fractional shrub/tree cover in a mire environment.” International Journal of Remote Sensing, 29 (5): 1261-1276.

[18]Wenzel, K., Rothermel, M., Fritsch, D., and Haala, N, 2013. "Image Acquisition and Model Selection for Multi-View Stereo." paper presented at Int. Arch. Photogramm. Remote Sens. Spatial Inf. Sci., XL-5/W1, 251-258, doi: 10.5194/isprsarchives-XL-5-W1-251-2013.

[19]Zhang Li, 2005. "Automatic Digital Surface Model (DSM) Generation from Linear Array Images." Ph D. diss., Swiss Federal Institute of Technology Zurich for the degree of Doctor of Technical Sciences.

[20]Zhu Qing. 2009. "The Study and Realization of Multi-view Image-based 3D Reconstruction Algorithm." Dissertation., University of Lanzhou. 